\title{
Heterostructures go down to the wire
}

\author{
The layered two-dimensional materials known as van der Waals heterostructures offer a versatile platform for the \\ creation of novel devices.
}

ince Konstantin Novoselov, Andre Geim and colleagues first reported ${ }^{1}$ work on the electronic properties of an atomically-thin slice of carbon atoms back in 2004, research into graphene and other twodimensional materials has expanded rapidly. Today, single-layer materials with an array of electronic behaviours are available. There is, to start, the semiconductor molybdenum disulfide, the insulator hexagonal boron nitride, and the ferromagnet chromium triiodide, not to mention graphene itself, which is a semimetal. But the capabilities and potential applications of such materials have also been notably extended by the development of the layered structures known as van der Waals heterostructures.

In van der Waals heterostructures, different two-dimensional materials are stacked one on top of the other. And thus, designer structures can be created by combining specifically chosen twodimensional materials. The approach has been used to build a variety of devices, from promising transistors ${ }^{2}$ and robust memristors ${ }^{3}$ to waveguide-integrated photodetectors ${ }^{4}$ and infrared hyperbolic metasurfaces ${ }^{5}$. But technical and conceptual innovations are still required in order develop more practical systems. In this issue of Nature Electronics, developments in both directions are reported.

Doping semiconductors by introducing chemical impurities and creating an excess of free electrons or holes is central to controlling the electronic properties of the materials and building commercial siliconbased electronics. But in two-dimensional materials such doping is difficult to achieve due the atomically thin crystal structures. In an Article in this issue, Alex Zettl and colleagues show that an electron beam can be used to write doping patterns in graphene and molybdenum disulfide van der Waals heterostructures.

The researchers - who are based at the University of California, Berkeley;

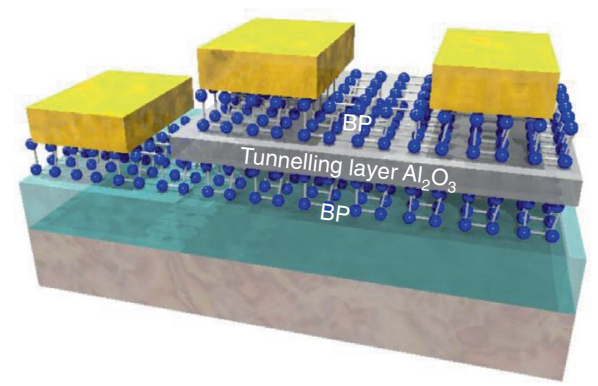

Schematic illustration of a transverse tunnelling field-effect transistor made from a van der Waals heterostructure. The heterostructure is composed of a layer of aluminium oxide $\left(\mathrm{Al}_{2} \mathrm{O}_{3}\right)$ sandwiched between layers of black phosphorus (BP). Figure adapted with permission from ref. ${ }^{8}$, Springer Nature Ltd.

Lawrence Berkeley National Laboratory; and the National Institute for Materials Science, Tsukuba - used heterostructures in which the active graphene or molybdenum disulfide layer is encapsulated in hexagonal boron nitride. The boron nitride protects the active two-dimensional material from direct exposure to the high-energy electron beam, while also enabling doping. In particular, the electron beam can activate charged defects in the boron nitride, which electrostatically dope the graphene or molybdenum disulfide. This doping is additionally controlled using a back-gate voltage. With the approach, Zettl and colleagues create high-resolution doping patterns and highquality $\mathrm{p}-\mathrm{n}$ junctions.

In another Article elsewhere in this issue, Yanqing $\mathrm{Wu}$ and colleagues at Huazhong University of Science and Technology and Peking University show that van der Waals heterostructures can be used to create unusual tunnel field-effect transistors in which the tunnelling current is in the transverse direction with respect to the drive current. The approach uses heterostructures composed of a layer of aluminium oxide sandwiched between layers of black phosphorus, and, due to an electrostatic effect, it can provide abrupt switching and a negative differential resistance.

Before graphene in the world of carbon nanostructures, there were carbon nanotubes (and before that, $\mathrm{C}_{60}$ ). But creating one-dimensional analogues of twodimensional van der Waals heterostructures has proved tricky. Such heterostructures were, however, recently developed by growing (via chemical vapour deposition) single-crystal layers of hexagonal boron nitride and molybdenum disulfide on single-walled carbon nanotube cores $^{6}$ (see also our Research Highlight on the work).

In related work, Peide Ye and colleagues have just reported in Nature Electronics that one-dimensional tellurium nanowires can be grown inside carbon and boron nitride nanotubes ${ }^{7}$. The researchers - who are based at Purdue University, the Washington University in St Louis, the University of Texas at Dallas and Michigan Technological University - show that van der Waals tellurium nanowires down to a single atomic chain can be isolated inside the nanotubes. They probe the electronic properties of the nanowires encapsulated in boron nitride nanotubes and also use the structures to build field-effect transistors that have a diameter of only $2 \mathrm{~nm}$.

Published online: 24 February 2020 https://doi.org/10.1038/s41928-020-0381-4

References

1. Novoselov, K. S. et al. Science 306, 666-669 (2004).

2. Iannaccone, G., Bonaccorso, F., Colombo, L. \& Fiori, G. Nat. Nanotechnol 13, 183-191 (2018).

3. Wang, M. et al. Nat. Electron 1, 130-136 (2018).

4. Flöry, N. et al. Nat. Nanotechnol 15, 118-124 (2020).

5. Li, P. et al. Science 359, 892-896 (2018)

6. Xiang, R. et al. Science 367, 537-542 (2020).

7. Qin, J.-K. et al. Nat. Electron. https://doi.org/10.1038/s41928-0200365-4 (2020).

8. Xiong, X. et al. Nat. Electron. https://doi.org/10.1038/s41928-0190364-5 (2020). 